\title{
Magellan Echelle Spectroscopy of TW Hydrae Brown Dwarfs
}

\author{
Subhanjoy Mohanty \\ Harvard-Smithsonian Center for Astrophysics, Cambridge, MA 02138, USA. \\ Ray Jayawardhana \\ Department of Astronomy, University of Michigan, Ann Arbor, MI 48109, USA. \\ and \\ David Barrado y Navascués \\ Laboratorio de Astofísica Espacial y Física Fundamental, INTA, 28080 Madrid, Spain.
}

\begin{abstract}
We present high-resolution optical spectroscopy of four candidate members of the nearby TW Hydrae young association including three brown dwarfs (2MASS 1207-3932, 2MASS 1139-3159 and TWA 5B) and one T Tauri multiple star (TWA 5A). Using echelle spectra from the Magellan Baade 6.5-meter telescope, we confirm the pre-main sequence status and cluster membership of the substellar candidates, through the detection of LiI, NaI consistent with low gravity, and radial velocity. Given their late spectral type $(\sim \mathrm{M} 8)$ and the youth of the association (age $\sim 10 \mathrm{Myr}$ ), cluster membership certifies these three objects as very low-mass young brown dwarfs. One of them (2MASS 1207-3932) shows strong emission both in the Hydrogen Balmer series $(\mathrm{H} \alpha$ to $\mathrm{H} \epsilon)$ and in $\mathrm{HeI}(4471,5876,6678$ and $7065 \AA$ ), compared to other young brown dwarfs of similar spectral type. The $\mathrm{H} \alpha$ line is also relatively broad (10\% width $\left.\sim 200 \mathrm{~km} \mathrm{~s}^{-1}\right)$ and asymmetric. These characteristics suggest that 2MASS 1207-3932 is a (weak) accretor. While we cannot rule out activity, comparison to a flaring field dwarf implies that such activity would have to be quite anomalous. The verification of accretion would make 2MASS 1207-3932 the oldest actively accreting brown dwarf known to date, suggesting that inner disk lifetimes in substellar objects can be comparable to those in stars, consistent with a similar formation mechanism. Finally, TWA 5A appears to be a variable accretor: observations separated by two days show broad accretion-like $\mathrm{H} \alpha$ (10\% width $\sim 270 \mathrm{~km} \mathrm{~s}^{-1}$ ), with significant changes in the $\mathrm{H} \alpha$ profile, as well as in the strengths of HeI, Na D and [OI6300]. TWA 5A is known to be a close triple; thus, our result implies that long-lived disks can exist even in multiple systems.
\end{abstract}


Subject headings: stars: low mass, brown dwarfs — stars: pre-main-sequence circumstellar matter — open clusters and associations: individual (TW Hydrae)

\section{Introduction}

The recent identification of several groups of young stars within 100 parsecs of the Sun has generated widespread interest (Jayawardhana \& Greene 2001). Given their proximity and age differences, these groups are ideally suited for detailed studies of the origin and early evolution of stars, brown dwarfs (BDs) and planets. Perhaps the most intensely studied among these groups is the TW Hydrae Association (TWA), which consists of $~ 20$ co-moving stars (Zuckerman et al. 2001) at a distance of 47-67 pc and dispersed over some 20 degrees on the sky. The members are mostly late-type ( $\mathrm{K}$ and $\mathrm{M})$ stars, and include several interesting

multiple systems (Brandeker, Jayawardhana \& Najita 2003, BJN03 hereafter, and references therein) and one A star. At an age of $~ 10 \mathrm{Myr}$, the TWA fills a significant gap in the age sequence between $\sim 1-$ Myr-old $\mathrm{T}$ Tauri stars in molecular clouds like Taurus-Auriga and the $~ 50$-Myr-old open clusters such as IC 2391. That is particularly useful for deriving strong constraints on disk evolution timescales. Their diverse disk properties suggest that the TWA stars are at an age when disks are rapidly evolving, through coagulation of dust and dissipation of gas (Jayawardhana et al. 1999a; 1999b).

Lowrance et al. (1999) found a BD candidate $\sim 2^{\prime \prime}$ from TWA 5A (CD $-33^{\circ} 7795$ ); TWA 5A and 5B are now confirmed as a common proper motion pair (BJN03 and references therein). Recently, Gizis (2002) reported two isolated substellar candidates from the 2-Micron All-Sky Survey that may be members of the TWA. Together, these three objects constitute a unique sample to explore the evolution of BD characteristics on a 10-Myr timescale. Here we report high-resolution optical spectroscopy that confirm the youth, group membership, and substellar status of these three objects. We also present a spectrum of the T Tauri multiple system TWA 5A, to which TWA 5B is bound. We use these spectra to investigate accretion, rotation and chromospheric activity.

\section{Observations \& Analysis}

We obtained high resolution optical spectra using the Magellan Inamori Kyocera Echelle spectrograph (Bernstein et al. 2002) on the Baade 6.5-meter telescope at Las Campanas Observatory, Chile in 2003 May. Consecutive spectra were obtained for the three BD candidate members: 3×1200s for 2MASS 1207-3932 (May 8), 3×1500s for 2MASS 1139-2649 (May 9), 
and $2 \times 1800$ s for TWA 5B (May 10). Additionally, we obtained two 600s exposures of TWA $5 \mathrm{~A}$, one each on May 8 and 10. The spectra of the 2MASS objects (from now on, 2M1207 and 2M1139) and the May 8 spectrum of TWA 5A were taken with a $1^{\prime \prime}$-wide by $5^{\prime \prime}$-long slit. The May 10 spectra of TWA 5A and 5B were obtained with a narrower $0.7^{\prime \prime} \times 5^{\prime \prime}$ slit. The separation between TWA $5 \mathrm{~A}$ and $5 \mathrm{~B}$ is $\sim 2^{\prime \prime}$, and TWA $5 \mathrm{~B}$ is $\sim 7$ magnitudes fainter in the optical. To ensure no contamination of the $5 \mathrm{~B}$ spectrum by $5 \mathrm{~A}$, therefore, we observed $5 \mathrm{~B}$ with a narrower slit $\left(0.7^{\prime \prime}\right)$, under optimal seeing conditions (better than $0.5^{\prime \prime}$ ), with the slit positioned roughly perpendicular to the 5A-5B axis. The coverage obtained was $\sim 3200-$ $4800 \AA$ in the blue, and $\sim 4800-8800 \AA$ in the red, with overlapping orders. The spectra are unbinned in wavelength, and binned by 2 pixels in the spatial direction. The $1^{\prime \prime}$ slit yielded a spectral resolution of $R \sim 19000$ in the red, and 25000 in the blue; the $0.7^{\prime \prime}$ slit resulted in $R \sim 27000$ and 36000 respectively. The data were reduced in standard fashion using IDL routines. We derive rotational velocities $(v \sin i)$ for our targets by cross-correlating with a 'spun-up' template of a slowly rotating standard. We used a combination of dwarf and giant spectra for the template (see Mohanty \& Basri 2003). Radial velocities $\left(\mathrm{v}_{\text {rad }}\right)$ were found by cross-correlating against the M6 dwarf Gl $406\left(\mathrm{v}_{\text {rad }} \approx 19 \mathrm{~km} \mathrm{~s}^{-1}\right)$. The $v \sin i$ and $\mathrm{v}_{\text {rad }}$ are listed in Table 1.

\section{Results \& Discussion}

\subsection{Membership \& Substellar Status}

All three TWA BD candidates have spectral types of M8-M8.5 (Gizis 2002; Webb et al. 1999), consistent with the features in our high-resolution data (e.g., strong TiO bands). In

all three, we detect LiI $6708 \AA$. They also exhibit narrow NaI $(\sim 8200 \AA)$ absorption profiles indicative of low gravity (intermediate between giants and dwarfs), and strong dMe-like $H \alpha$ emission (Fig. 1; equivalent widths -EW- for $H \alpha$ and LiI given in Table 1). In concert, these facts confirm the Pre-Main Sequence (PMS) status of TWA 5B, 2M1207 and 2M1139: we can confidently exclude field M dwarfs (no LiI), low-mass field BDs with undepleted Li (type $\sim$ L2 or later; gravity $\gtrsim$ dwarfs values), Li-rich giants (much lower gravity; $H \alpha$ in absorption) and sub-giants (MS lifetime of M dwarfs too long for M-type subgiants to have formed yet).

For all three objects, our derived $\mathrm{v}_{\text {rad }}$ are commensurate with those found for other, bona-fide members of the association (Torres et al. 2003). TWA 5B is also confirmed as a common proper motion companion to TWA 5A (BJN03 and references therein). Gizis (2002) has found a proper motion consistent with membership for 2M1207. In conjunction with our $\mathrm{v}_{\text {rad }}$ measurements and confirmation of PMS status, these observations verify 
TWA membership for both TWA 5B and 2M1207. For 2M1139, Gizis (2002) found a proper motion apparently inconsistent with membership. However, he notes that the astrometric uncertainty is large. Therefore, given its PMS status and a $\mathrm{v}_{\text {rad }}$ consistent with other members, we consider it likely that 2M1139 also belongs to the TWA. More precise astrometric measurents are required to verify this.

All objects with detected Lithium and $\mathrm{T}_{\text {eff }}$ less than about $2800 \mathrm{~K}$ are expected to be substellar, regardless of age (see Basri 2000). The M8-8.5 spectral type of 2M1207, 2M1139 and TWA 5B, which implies $\mathrm{T}_{\text {eff }}<2800 \mathrm{~K}$ (e.g., Luhman 1999), therefore ensures that they are BDs, since they all show Li. An age is required, however, to derive a mass. Since we confirm association membership for at least 2M1207 and TWA 5B, an age of $\sim 10$ Myr is justified for them. Comparing to the evolutionary tracks of Chabrier et al. (2000) then yields $\sim 35$ Jupiters for both. The same value is obtained for 2M1139, if it too is a member. Even if it is not, one can still use the fact of Li detection, and a $\mathrm{T}_{\text {eff }}$ estimate $(\sim 2700 \mathrm{~K}$; Luhman 1999), to put upper limits on mass and age of $\sim 65$ Jupiters and $\sim 250$ Myr respectively, using the same models.

\subsection{Accretion}

At very low masses and weak accretion rates, usual accretion indicators (e.g., Stark broadening of $H \alpha$ wings, emission in OI and CaII) may disappear. Simultaneously, other lines commonly associated with accretion at higher masses (e.g., HeI, upper Balmer lines) may appear in non-accreting, chromospherically active low-mass objects due to the lowering of the photospheric continuum with decreasing $T_{\text {eff }}$ (e.g., Jayawardhana, Mohanty, \& Basri 2003, hereafter JMB03). Consequently, it is difficult to distinguish between accretion and activity in weakly accreting low-mass objects.

Here we conservatively identify probable accretors based on asymmetric and broad $H \alpha$ ( $10 \%$ width $\gtrsim 200 \mathrm{~km} \mathrm{~s}^{-1}$, following JMB03) and the detection of usual indicators of accretion at larger masses, such as HeI and upper Balmer lines, at a level higher than the average for a given spectral type (the same criteria adopted by Muzerolle et al. 2003). By these conditions, 2M1139 and TWA 5B are chromospherically active, but not accreting. 2M1207 and TWA 5A, however, do appear to be accretors, as discussed below.

2MASS 120\%-3932: As Fig. 2 shows, significant emission is seen in HeI and in the upper Balmer series lines upto $\mathrm{H} \epsilon$ in this object. A perusal of the recent study by Muzerolle et al. (2003), meanwhile, shows that non-accreting M7-8.5 objects in their sample usually do not show HeI (at $6678 \AA$, the only HeI line they include), or Balmer lines beyond $H \beta$. 
Next, in Fig. 3, we show the normalized $H \alpha$ profiles from our 3 consecutive spectra of 2M1207. A clear asymmetry is seen in the first and third profiles, with much enhanced emission blueward of line-center. In the second profile, the full-width at $10 \%$ of peak flux is $\sim 200 \mathrm{~km} \mathrm{~s}^{-1}$ : not unreasonable for very low-mass, low accretion-rate objects, and similar to that seen by JMB03 in a couple of mid- to late M accretors. In the first and last profiles, the width is slightly less, at $\sim 170 \mathrm{~km} \mathrm{~s}^{-1}$. However, superimposing the three spectra (not shown) reveals that the line wings are exactly the same; the smaller $10 \%$ width in the first and last is artificially induced simply by the increase in peak flux. We therefore adopt $\sim 200 \mathrm{~km} \mathrm{~s}^{-1}$ as the more robust value. By all our criteria, therefore, 2M1207 appears to be (weakly) accreting. Can the emission in this object simply be a result of enhanced activity compared to other, similar spectral type objects? While we cannot rule this out, the following argument makes this seem unlikely. In one of our high-resolution spectra of the field M7.5 dwarf LHS 2397A, taken during a flare, the $H \alpha \mathrm{EW}$ is $\sim 30 \AA$ (Mohanty \& Basri 2003), i.e., almost exactly the same as observed in 2M1207. If the emission in 2M1207 were due to chromospheric activity, we would expect its line profiles to look very similar to those of LHS 2397A. given the nearly identical spectral types. The line profile comparison is shown in Fig.3. Clearly, the LHS 2397A $H \alpha$ profile much more symmetric compared to the first and last 2M1207 spectra, and significantly narrower in all three cases: 2M1207 exhibits broader $H \alpha$ line wings, as expected from accretion. The HeI line at $6678 \AA$ is also much stronger in 2M1207 than in LHS 2397A; since the spectral types (and hence the underlying continuum) are almost the same, the actual flux in HeI emission is thus also higher. This too indicates that the same physical process is not responsible for the emission in the two objects; in particular, excess HeI emission can arise from the very hot accretion-shock region. We suggest, therefore, that 2M1207 is a bona-fide accretor. However, our arguments against activity are not ironclad, and the presence of accretion needs to be checked through other diagnostics. The lack of a $K-L^{\prime}$ excess in 2M1207 (Jayawardhana et al. 2003) could be the result of a nearly edge-on disk inclination or grain growth.

If accretion in 2M1207 is confirmed, it would suggest that inner disk lifetimes of substellar objects can be comparable to those of their stellar counterparts, and further strengthen the case for a common formation mechanism for BDs and stars.

TWA 5A: The $10 \%$ full-width of the $H \alpha$ line in TWA $5 \mathrm{~A}$ is $\sim 270 \mathrm{~km} \mathrm{~s}^{-1}$, which is commensurate with accretion (e.g., JMB03). However, this object is known to be a triple, with $\mathrm{a} \sim 0$ ". 06 binary resolved by adaptive optics (BJN03) as well as a spectroscopic companion (Torres et al. 2003). To discount the possibility that the $H \alpha$ line is broadened to accretion-like widths simply due to blending of $H \alpha$ from the three stars, we compare our two spectra of TWA 5A, obtained two days apart. Fig. 4 shows that the spectra are exactly the same, except in lines that are accretion indicators. This implies that the spectral change 
is not due to variations in line-blending, but in the intrinsic parameters of (at least) one of the TWA 5A components. In particular, we see that the first spectrum (in black) shows excess red-shifted emission and blue-shifted absorption in $H \alpha$, HeI5876 $\AA$ and $\mathrm{Na} \mathrm{D}$, as well as excess emission in [OI6300], compared to the second spectrum (in grey). Though $H \alpha$, $\mathrm{NaD}$ and HeI emission may conceivably arise from chromospheric activity, the blue-shifted absorption seen in these three lines strongly suggests accretion. Furthermore, [OI6300] is an excellent diagnostic of outflowing winds associated with accretion; it is often seen in accreting CTTs, but never in non-accreting WTTs (Muzerolle et al. 2003). All this supports ongoing accretion in the TWA 5A system. The fact that the two spectra differ significantly in the accretion diagnostics implies that the process is variable. Our results also suggest that inner disks can be long-lived even in close multiple systems.

\subsection{Rotation \& Activity}

In field dwarfs down to $\sim$ M8, a saturation-type rotation-activity connection is seen: those rotating faster than $\sim 5 \mathrm{~km} \mathrm{~s}^{-1}$ exhibit saturated levels of chromospheric $H \alpha$ emission, while slower rotators show a range of smaller $H \alpha$ fluxes (Mohanty \& Basri 2003). For our young sample, we will discuss this issue in more detail in a future paper, after deriving accurate $\mathrm{T}_{\text {eff }}$ in order to convert the observed $H \alpha$ EWs to fluxes. However, we note here that the $H \alpha$ EW observed in our three M8 PMS objects are, at the very least, comparable to those seen in saturated field dwarfs of the same spectral type; this is in keeping with their moderately rapid rotation $\left(>10 \mathrm{~km} \mathrm{~s}^{-1}\right)$. In $2 \mathrm{M} 1207$, the $H \alpha$ emission is far stronger than in merely saturated field dwarfs; as discussed above, the EW in this case is comparable to that in strongly flaring M8 dwarfs. Similar EWs have been seen in some other young, (apparently) non-accreting late-M objects, lending support to the idea that activity may be enhanced in young low-mass objects compared to field objects of similar spectral type (e.g., Jayawardhana, Mohanty, \& Basri 2002, 2003). However, the $H \alpha$ profile in these cases is narrow and symmetric. If $2 \mathrm{M} 1207$ were a non-accretor, then the strong asymmetries seen in at least two of its $H \alpha$ profiles would be puzzling.

We are most grateful to Gibor Basri for insightful discussions. We thank the referee Jeff Valenti for a prompt review and the Magellan staff for their assistance. S.M. is indebted to the SIM-YSO group for the postdoctoral fellowship that made this research possible. This work was supported in part by NSF grant AST-0205130 and NASA grant NAG5-11905 to R.J. 


\section{REFERENCES}

Basri, G. 2000, ARA\&A, 38, 485

Bernstein, R.A., et al. 2002, Proc. SPIE 4841, 1694

Brandeker, A., Jayawardhana, R. \& Najita, J. 2003, AJ, in press [BJN03]

Chabrier, G., et al. 2000, ApJ, 542, 464

Gizis, J.E. 2002, ApJ, 575, 484

Jayawardhana, R. et al. 1999a, ApJ, 520, L41

Jayawardhana, R. et al. 1999b, ApJ, 521, L129

Jayawardhana, R. \& Greene, T.P. (ed.) 2001, Young Stars Near Earth: Progress and Prospects, ASP Conf. Ser. 244 (San Fransisco: ASP)

Jayawardhana, R., Mohanty, S., \& Basri, G. 2002, ApJ, 578, L141

Jayawardhana, R., Mohanty, S., \& Basri, G. 2003, ApJ, in press [JMB03]

Jayawardhana, R., Ardila, D.R., Stelzer, B., \& Haisch, K.E., Jr. 2003, AJ, in press

Lowrance, P.J., et al. 1999, ApJ, 512, L69

Luhman, K. 1999, ApJ, 525, 466

Mohanty, S., \& Basri, G. 2003, ApJ, 583, 451

Muzerolle, J., et al. 2003, ApJ, in press

Torres, G., et al. 2003, AJ, 125, 825

Webb, R.A., et al. 1999, ApJ, 512, L63

Zuckerman, B., Webb, R.A., Schwartz, M., \& Becklin, E.E. 2001, ApJ, 549, L233 

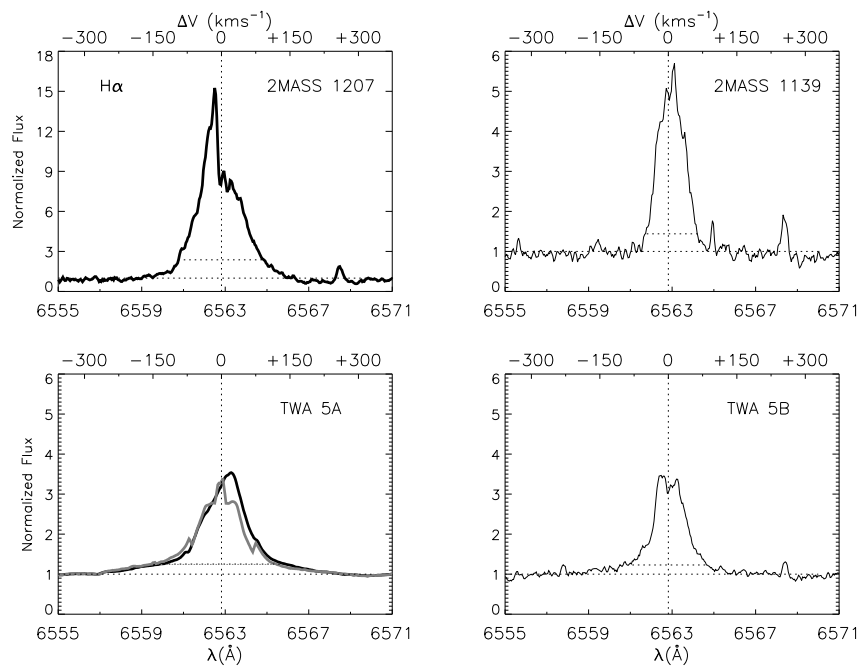

Fig. 1. - Averaged $H \alpha$ profiles for our targets (both profiles shown for TWA 5A). Thick lines indicate the two probable accretors. The horizontal dashed lines indicate the continuum and $10 \%$ of peak emission. All profiles are shifted to zero-velocity (approximate for TWA $5 \mathrm{~A}$; we have not calculated its $\mathrm{v}_{\text {rad }}$ ).
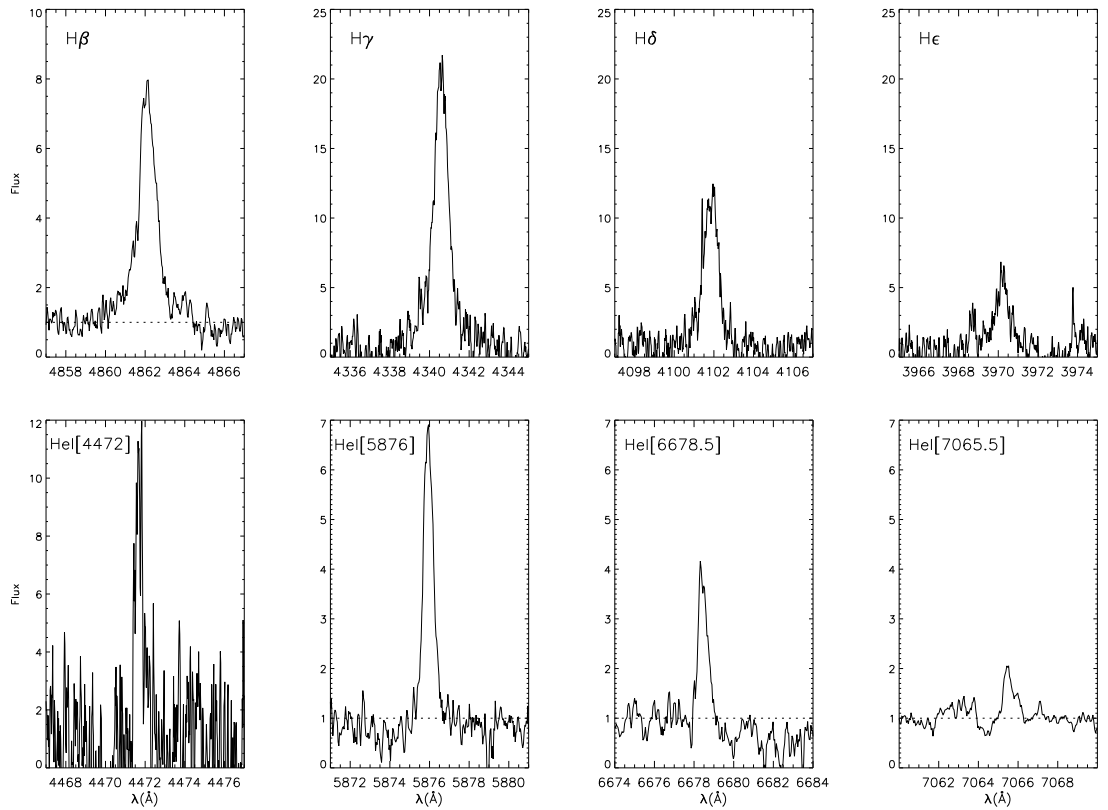

Fig. 2.- Averaged HI and HeI emission in 2MASS 1207. A continuum was detected around only some of these lines; these have been continuum-normalized (continuum: dashed horizontal line). In the others, no true continuum was detected, making flux or EW measurements impossible; these have only been divided by the r.m.s. of the surrounding noise for clarity. 

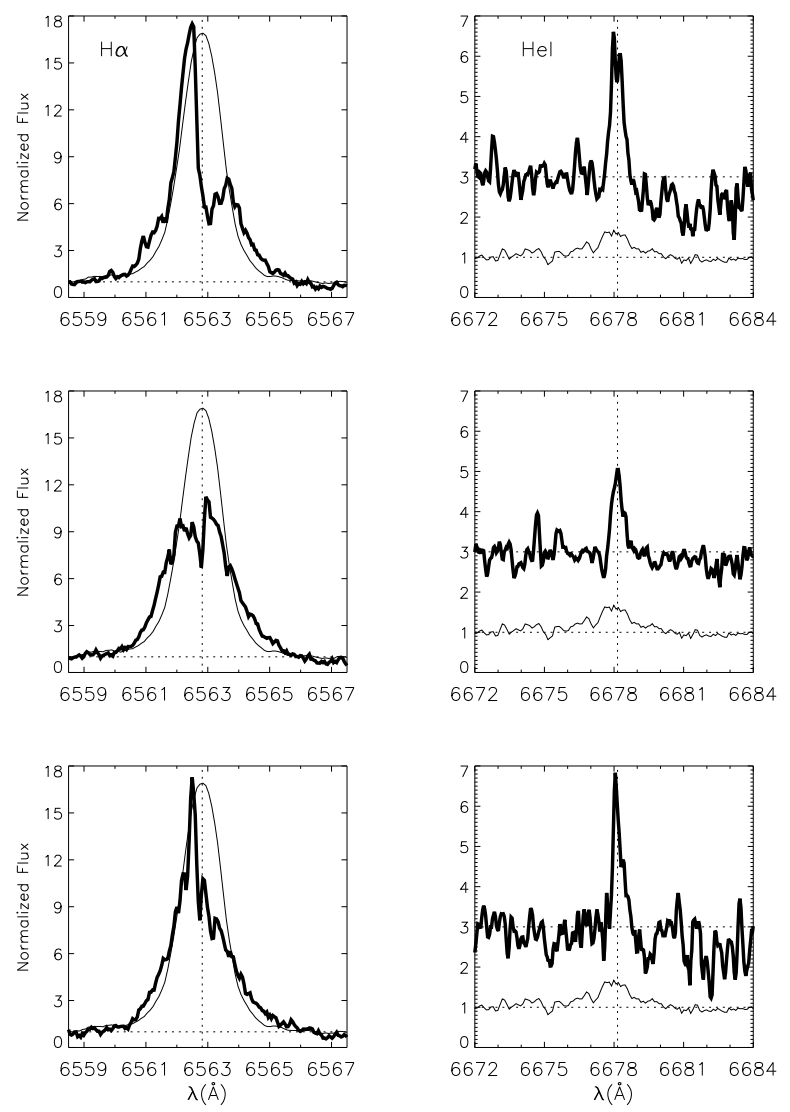

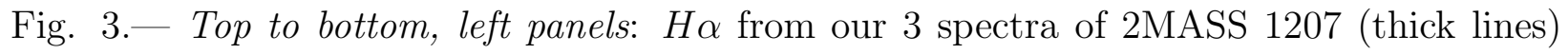
compared to the $H \alpha$ in the flaring field M7.5 dwarf LHS 2397A (observed at similar resolution on Keck HIRES). Top to bottom, right panels: HeI6678̊ comparison. 

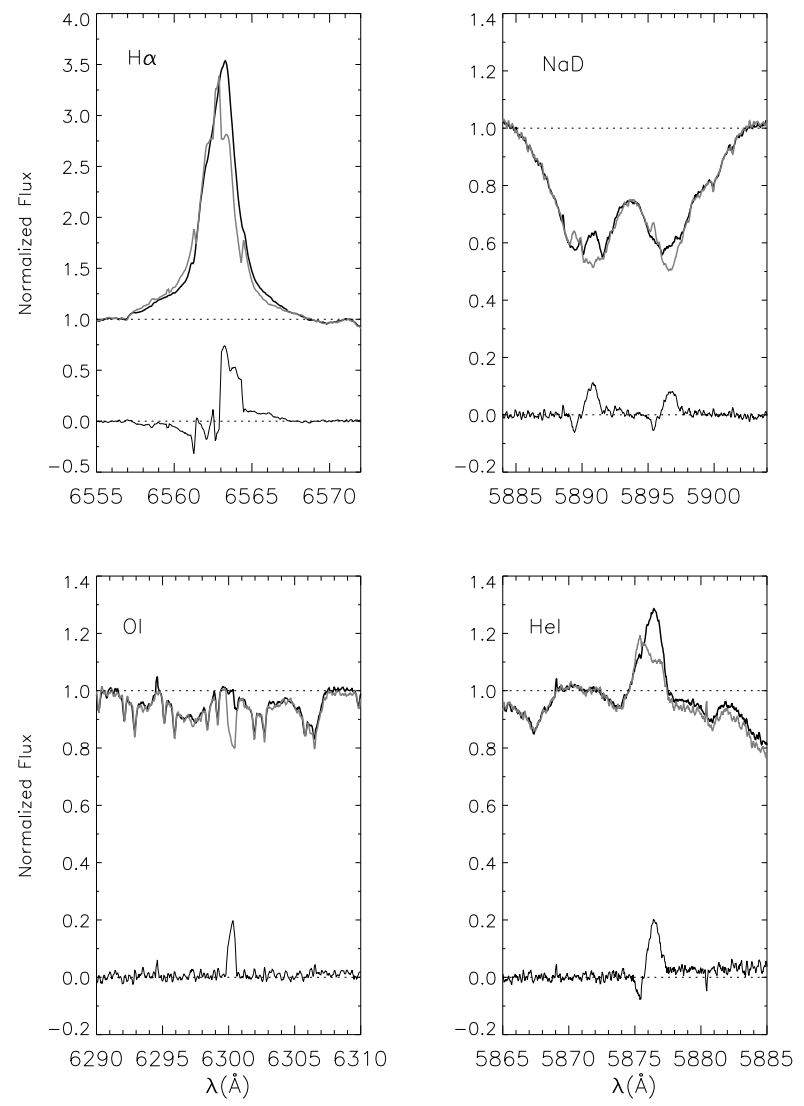

Fig. 4.- Comparison of two TWA 5A spectra, in regions containing accretion-indicating

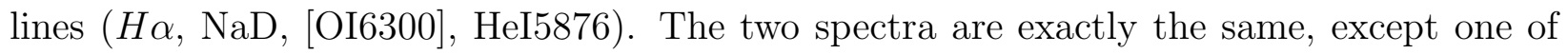
them (black) shows excess emission in $H \alpha$, NaD, OI \& HeI, and blue-shifted absorption in $H \alpha, \mathrm{NaD} \& \mathrm{HeI}$, compared to the other spectrum (grey). 
Table 1. Spectroscopic Properties of TWA Targets

\begin{tabular}{lcccccc}
\hline \hline \multicolumn{1}{c}{ Object } & Sp Type $^{\mathrm{a}}$ & $\begin{array}{c}\mathrm{v}_{\text {rad }} \\
\left(\mathrm{km} \mathrm{s}^{-1}\right)\end{array}$ & $\begin{array}{c}v \sin i \\
\left(\mathrm{~km} \mathrm{~s}^{-1}\right)\end{array}$ & $\begin{array}{c}\mathrm{LiI} \mathrm{EW}^{\mathrm{b}} \\
(\AA)\end{array}$ & $\begin{array}{c}\mathrm{H} \alpha \mathrm{EW}^{\mathrm{b}} \\
(\AA)\end{array}$ & $\begin{array}{c}\mathrm{H} \alpha 10 \% \mathrm{FW}^{\mathrm{c}} \\
\left(\mathrm{km} \mathrm{s}^{-1}\right)\end{array}$ \\
\hline 2MASS 1207-3932 & $\mathrm{M} 8$ & $11.2 \pm 2$ & $13 \pm 2$ & 0.5 & 27.7 & $204 \pm 10$ \\
2MASS 1139-3159 & $\mathrm{M} 8$ & $11.6 \pm 2$ & $25 \pm 2$ & 0.5 & 7.3 & $111 \pm 10$ \\
TWA 5B & $\sim \mathrm{M} 8.5$ & $13.4 \pm 2$ & $16 \pm 2$ & 0.3 & 5.1 & $162 \pm 10$ \\
TWA 5A & $\mathrm{M} 1.5$ & - & - & 0.6 & $7.2 / 6.6^{\mathrm{e}}$ & $266 \pm 10$ \\
\hline
\end{tabular}

a Spectral types for 2MASS objects from Gizis (2002) and for TWA 5A \& 5B from Webb et al. (1999).

${ }^{\mathrm{b}}$ Pseudo-equivalent widths, for LiI \& $H \alpha$; estimated $H \alpha$ EW errors $\lesssim 10 \%$.

${ }^{\mathrm{c}}$ For 2M1139 \& TWA 5B, we quote the average width from their consecutive spectra over a single night. For $2 \mathrm{M} 1207$, we adopt the $H \alpha 10 \%$ width of only the second spectrum as the realistic value (see text); the average width from all three spectra is $187 \pm 10 \mathrm{~km} \mathrm{~s}^{-1}$. For TWA 5A, we quote $10 \% \mathrm{FW}$ values for both nights; they are very similar.

${ }^{\mathrm{d}}$ We did not derive $\mathrm{v}_{\text {rad }}$ or $v \sin i$ for the TWA 5A system, given its known $\mathrm{v}_{\text {rad }}$ variability (Torres et al. 2003), and the questionable accuracy of $v \sin i$ given possible line blending.

${ }^{\mathrm{e}} \mathrm{In}$ TWA $5 \mathrm{~A}$, the larger $H \alpha \mathrm{EW}$ is for the spectrum with excess accretion-related emission. 